\title{
Debating Frontline Therapy in Chronic Myeloid Leukemia
}

\author{
$\mathrm{Xia} \mathrm{Bi}^{*+}$, Sabarina Ramanathan ${ }^{\dagger}$ and Gina Keiffer \\ Department of Medical Oncology, Thomas Jefferson University, Philadelphia, PA, United States
}

Keywords: CML - chronic myelogenous leukemia, imatinib, second generation tyrosine kinase inhibitors, TKI tyrosine kinase inhibitor, dasatinib, nilotinib, bosutinib, ponatinib

\section{INTRODUCTION}

The development of tyrosine kinase inhibitors (TKIs) in the early 2000s revolutionized the therapeutic landscape for chronic phase (CP) chronic myeloid leukemia (CML). Interferon alpha was previously the standard treatment for patients with CP CML prior to the development of TKIs; however, survival was dismal, with a median of 5-6 years (1). With the advent of TKI therapy, the estimated 10-year overall survival increased from less than $20 \%$ to more than $80 \%$ (2). When optimal therapy is instituted with appropriate monitoring, patients with CP CML now live close to normal life spans $(3,4)$.

Imatinib, the first of these revolutionary medications, was approved by the FDA for the treatment of CML after failure of interferon-alpha therapy in 2001 and for newly diagnosed CP CML patients in 2003. Shortly thereafter, following preclinical and clinical data demonstrating increased potency against the BCR-ABL target, frontline approvals for the second-generation TKIs (dasatinib, nilotinib, and bosutinib) soon followed based on the results of their respective clinical trials (DASISION, ENEST and BFORE) (5-7). Each of these trials compared the efficacy of imatinib $400 \mathrm{mg}$ daily with the corresponding second-generation TKI. Notably, no significant survival difference has been demonstrated between imatinib and any of the second-generation inhibitors. Accordingly, the European Society for Medical Oncology (ESMO) and the National Comprehensive Cancer Network (NCCN) practice guidelines recommend choosing a therapeutic agent based on risk scoring, age, and comorbidities $(8,9)$. Thus, the optimal frontline treatment of CP CML has become the subject of debate.

A recently published meta-analysis sought to answer this question by comparing the safety and efficacy of imatinib versus dasatinib, nilotinib, bosutinib and ponatinib for initial treatment of CP CML (10). Second- and third-generation TKIs demonstrated superior clinical outcomes but also increased toxicity. The authors concluded that the choice of frontline therapy should depend on patients' age and comorbidities. They suggested that patients without comorbidities should receive second-generation TKIs as initial therapy and that imatinib should be the preferred initial therapy for older patients or those with comorbidities. Notably, third-generation TKI (ponatinib) has not been approved nor is recommended as the frontline treatment of CP AML. Herein, we will review this recently published work and present the arguments for and against the use of secondgeneration TKIs as initial therapy for CP CML. 


\section{THE DATA}

In this meta-analysis, the authors systematically reviewed randomized controlled trials (RCTs) that compared the efficacy and safety of imatinib $v s$ second-generation (dasatinib, nilotinib, bosutinib) or third-generation (ponatinib) TKIs in adults with newly diagnosed Philadelphia chromosome-positive $(\mathrm{Ph}+) \mathrm{CP}$ CML. Nineteen relevant studies and 15 relevant abstracts published between 1990 and 2019, corresponding to 7 RCTs involving 3262 participants, were included. The primary outcomes were overall survival (OS) and progression-free survival (PFS). Secondary outcomes included various efficacy and safety measures.

There was no statistically significant difference in the primary outcomes, although only 2 of the 7 RCTs reported OS and PFS up to 60 months and only 1 reported OS up to 72 months. In terms of secondary outcomes, all of the pooled efficacy outcomes except for drug discontinuation showed a clear advantage of second- and third-generation TKIs over imatinib. Table 1 summarizes the relative risks (RRs) of later-generation TKIs in comparison with imatinib in terms of efficacy and toxicity as reported in the ENEST, DASISION, and BFORE studies and in the pooled analysis. The RR of major molecular response (MMR) after 3 months was statistically higher than all other efficacy outcomes in patients treated with later-generation TKIs (RR = $4.50 ; 95 \%$ confidence interval [CI], 2.23-9.09). In terms of adverse events, there were more cases of thrombocytopenia, cardiovascular events, pancreatic and hepatic effects in patients treated with later-generation TKIs. Specifically, hepatic effects had the highest RR in the bosutinib group.

\section{POINT: THE CASE FOR USING SECOND GENERATION TKIS AS INITIAL THERAPY FOR CP CML}

The meta-analysis demonstrated a statistically significant improvement of 3-month MMR and other efficacy outcomes in patients treated with second- and third-generation TKIs. Attainment of an early molecular response (EMR; BCR-ABL1 IS $\leq 10 \%$ at 3 months) has been shown to be an important treatment milestone in patients with newly diagnosed CP CML (11-13) and is the first benchmark for evaluating responses to TKI therapy $(9,14)$. Failure to achieve an EMR suggests treatment failure, and consideration should be given to alternate therapy $(9,15)$. Previous studies showed superior PFS and OS in patients who were able to achieve this early molecular milestone, and that EMR failure was associated with lower rates of molecular response and increased risk of disease progression (16-19). Therefore, achievement of an EMR may predict longterm clinical outcomes and allow early intervention for patients who are less likely to respond to treatment.

Another important consideration in selecting an initial therapy is the ability to safely stop therapy and maintain a treatment-free remission (TFR). TFR is defined as maintaining

TABLE 1 | Relative risk of later-generation TKls in comparison with imatinib by efficacy and safety endpoints.

\begin{tabular}{lccc}
\hline & $\begin{array}{c}\text { ENEST* } \\
\text { RR (95\% CI) }\end{array}$ & $\begin{array}{c}\text { DASISION } \\
\text { RR (95\% CI) }\end{array}$ & $\begin{array}{c}\text { BFORE } \\
\text { RR (95\% CI) }\end{array}$ \\
\hline RR (95\% CI)
\end{tabular}

EMR, early molecular response (Bcr-Ab/IS = 10\%); MMR, major molecular response (Bcr-Ab/IS = 0.1\%); CCyR, complete cytogenetic response; MR4, Bcr-Abl IS = 0.01\%; MR4.5, Bcr$A b /$ IS $=0.0032 \%$; $A P / B P$, accelerated phase/blast phase transformation.

$N R$, not reported; NE, not estimable.

Bold = statistically significant.

*Data for nilotinib 300mg twice daily. 
a BCR-ABL1 IS $\leq 0.1 \%$ (MMR) off TKI therapy. In order to safely discontinue TKI therapy, patients are recommended to achieve and maintain a deep molecular response (DMR; BCR-ABL1 IS $\leq 0.01 \%$ ) for $\geq 2$ years (9). TFR is most successful in patients with at least 4 years of TKI therapy who achieve and maintain DMR for at least 2 years prior to treatment cessation $(14,20,21)$. Successful TFR limits treatment-associated AEs, decreases cost and allows for fertility. Thus, the ability to maintain TFR is especially important for young patients and those who desire fertility.

In this meta-analysis, more patients treated with latergeneration TKIs were able to achieve a MR4 and MR4.5 (RR = $1.64, \mathrm{RR}=2.63$, respectively), which is associated with higher survival probabilities and greater chance of TFR (22). The ENEST study, with more than 10 years of follow up, demonstrated higher cumulative incidence of achieving MR4.5 for nilotinib-treated patients $(61 \%)$ than imatinib-treated patients (39.2\%) (23). Patients treated with nilotinib also had higher rates of TFR eligibility (48.6\% vs 29.7\%) (23), which supports the use of second-generation TKIs in patients aiming for TFR.

Treatment with later-generation TKIs is also associated with decreased progression to AP or $\mathrm{BP}(\mathrm{RR}=0.44,95 \% \mathrm{CI} 0.26$ 0.74 ), thus resulting in fewer patients needing intensive chemotherapy and stem cell transplantation and preventing the significant morbidity, mortality and cost associated with these therapies. This benefit was seen especially in patients with high risk Sokal scores, where only 7 (9\%) patients treated with nilotinib 300mg twice daily experienced progression to AP and BP compared to $11(14 \%)$ patients treated with imatinib in the ENEST study (24).

\section{COUNTERPOINT: THE CASE AGAINST USING SECOND GENERATION TKIS AS INITIAL THERAPY FOR CP CML}

Imatinib was the first TKI to be approved by the FDA for patients with CML in all phases based on the results of the landmark IRIS study which compared the efficacy of imatinib with the standard of care interferon and cytarabine. After a median follow-up of 19 months, imatinib demonstrated significant improvement in rates of CCyR (74\% versus $9 \%$, $\mathrm{P}<0.001)$ and freedom from progression to $\mathrm{AP}$ or $\mathrm{BP}$ at 12 months (99\% versus 93\%, $\mathrm{P}<0.001$ ) (25). Further follow-up at 10 years demonstrated that $93 \%$ of imatinib-treated patients achieved MMR, 63\% achieved MR4.5 and, astoundingly, overall survival was $83.3 \%$, establishing the long-term durability of imatinib (2).

Despite improvement the improvement in time to response and depth of response seen with second generation TKIs, they have never shown an overall survival or progression free survival benefit beyond imatinib. The 5-year OS rates for nilotinib vs imatinib was $94 \%$ vs $92 \%$, and dasatinib vs imatinib was $91 \%$ vs 90\% (Table 1) $(19,24,26)$. Outcomes stratified by disease risk score (Sokal, Euro/Hasford) similarly demonstrated no significant survival difference between nilotinib and imatinib (6). While the higher dose of nilotinib (400mg twice daily) showed superior OS and PFS, this dosage was associated with unacceptable levels of cardiovascular toxicity and is only employed in AP CML. Similarly, after 5 years of follow up of the BFORE trial, there was no differences in EFS in treatment arms and OS rates were comparable at $94.5 \%$ for bosutinib $v s$ 94.6\% for imatinib (27).

In addition to the lack of improvement in overall survival, second-generation TKIs have been shown to have increased toxicity compared to imatinib. All TKIs cause cytopenias and gastrointestinal (GI) side effects, but in clinical experience, most AEs for imatinib are mild and manageable while the newer agents portend greater early and later serious AEs and grade $\geq 3$ AEs that lead to treatment discontinuation. The risk of vasoocclusive events (VOEs) in particular is increased with secondgeneration TKIs. A meta-analysis pooling ten trials consisting of $>3000$ patients demonstrated that dasatinib, nilotinib, and ponatinib usage increased risk of vascular occlusive events. Events were observed in $5.8 \%$ of patients (93 of 1582) treated with second-generation TKIs $v s 1.0 \%$ of patients (13 of 1253) on imatinib. The study reported significantly higher VOEs with nilotinib (odds ratio [OR], 3.45) and dasatinib (OR, 3.86) in comparison to imatinib (28).

Finally, for any potentially life-long therapy, the overall cost of treatment, including not only the cost of the treatment itself but of the additional cost of managing adverse effects and monitoring of therapy, must be considered in selecting an upfront treatment strategy. Cost-effectiveness analyses in the US and Japan simulating the clinical course of 10 years of CML treatment, mapped cost estimate probabilities starting with imatinib, dasatinib, nilotinib, or any TKI according to the physician's choice. This model demonstrated a value advantage for imatinib-first sequential treatment strategies over the initial use of second-generation agents even after factoring in drug discontinuation at the 2-year DMR target (29). While the cost of imatinib has varied over the years, data suggests that generic imatinib could be purchased at a daily cost as low as \$20 in 2018. Interestingly, despite the lack of survival data, the use of secondgeneration TKIs tripled from 19\% to 56\% from the years 2010 to 2019. In parallel, the daily cost of newer agents increased from $\$ 243$ per day in 2010 to $\$ 354$ in 2018 validating that individual and overall health care costs are higher with newer agents potentially causing higher out-of-pocket expenses, increased financial burden and treatment delays (30).

While maintaining deep and durable molecular responses remains the therapeutic goal for patients with CML, the ultimate goal of therapy is to help patients live better and longer. To this end, imatinib has demonstrated prolonged survival over sufficient observation periods as well as improved tolerability compared to second generation TKIs. Additionally, imatinib is generic and is more widely available than second generation TKIs and, despite few patients attaining TFR, imatinib has shown improved cost effectiveness beyond second generation TKIs. It is worth noting that second generation TKIs remain a very effective second-line therapy for patients who progress on or 
are intolerant of imatinib, allowing almost half of those patients to achieve a complete cytogenetic response (31).

\section{CONCLUSIONS}

A first-generation TKI, imatinib and three second-generation TKIs, dasatinib, nilotinib, and bosutinib, have all been approved for the initial treatment of CP CML, prompting the question of which TKI is the best one to use as initial therapy. Although no additional OS or PFS benefit has been seen with the use of second-generation TKIs compared to imatinib, they have demonstrated superiority in several surrogate endpoints of clinical efficacy, including higher rates and depth of response and decreased progression into AP and BP. In the absence of an overall survival or progression free survival benefit, the decision for which TKI to use as initial therapy should take in to account individual patients' medical comorbidities and the importance of attaining TFR, particularly for patients who desire fertility.

We propose using second-generation TKIs as frontline therapy in young patients, those who desire fertility and those

\section{REFERENCES}

1. Hehlmann R, Heimpel H, Hasford J, Kolb HJ, Pralle H, Hossfeld DK, et al. Randomized Comparison of Interferon-Alpha With Busulfan and Hydroxyurea in Chronic Myelogenous Leukemia. The German CML Study Group. Blood (1994) 84(12):4064-77. doi: 10.1182/blood.V84.12.4064. bloodjournal84124064

2. Hochhaus A, Larson RA, Guilhot F, Radich JP, Branford S, Hughes TP, et al. Long-Term Outcomes of Imatinib Treatment for Chronic Myeloid Leukemia. N Engl J Med (2017) 376(10):917-27. doi: 10.1056/NEJMoa1609324

3. Druker BJ, Guilhot F, O'Brien SG, Gathmann I, Kantarjian H, Gattermann N, et al. Five-Year Follow-Up of Patients Receiving Imatinib for Chronic Myeloid Leukemia. N Engl J Med (2006) 355(23):2408-17. doi: 10.1056/ NEJMoa062867

4. Kalmanti L, Saussele S, Lauseker M, Muller MC, Dietz CT, Heinrich L, et al. Safety and Efficacy of Imatinib in CML Over a Period of 10 Years: Data From the Randomized CML-Study IV. Leukemia (2015) 29(5):1123-32. doi: 10.1038/leu.2015.36

5. Kantarjian H, Shah NP, Hochhaus A, Cortes J, Shah S, Ayala M, et al. Dasatinib Versus Imatinib in Newly Diagnosed Chronic-Phase Chronic Myeloid Leukemia. N Engl J Med (2010) 362(24):2260-70. doi: 10.1056/ NEJMoa1002315

6. Saglio G, Kim DW, Issaragrisil S, le Coutre P, Etienne G, Lobo C, et al. Nilotinib Versus Imatinib for Newly Diagnosed Chronic Myeloid Leukemia. N Engl J Med (2010) 362(24):2251-9. doi: 10.1056/NEJMoa0912614

7. Cortes JE, Kim DW, Kantarjian HM, Brummendorf TH, Dyagil I, Griskevicius L, et al. Bosutinib Versus Imatinib in Newly Diagnosed Chronic-Phase Chronic Myeloid Leukemia: Results From the BELA Trial. J Clin Oncol (2012) 30(28):3486-92. doi: 10.1200/JCO.2011.38.7522

8. Hochhaus A, Saussele S, Rosti G, Mahon FX, Janssen J, Hjorth-Hansen H, et al. Chronic Myeloid Leukaemia: ESMO Clinical Practice Guidelines for Diagnosis, Treatment and Follow-Up. Ann Oncol (2018) 29(Suppl 4):iv261. doi: 10.1093/annonc/mdy159

9. Deininger MW, Shah NP, Altman JK, Berman E, Bhatia R, Bhatnagar B, et al. Chronic Myeloid Leukemia, Version 2.2021, NCCN Clinical Practice Guidelines in Oncology. J Natl Compr Canc Netw (2020) 18(10):1385-415. doi: 10.6004/jnccn.2020.0047

10. Vener C, Banzi R, Ambrogi F, Ferrero A, Saglio G, Pravettoni G, et al. FirstLine Imatinib vs Second- and Third-Generation TKIs for Chronic-Phase with intermediate or high risk disease. Imatinib is more appropriate for low- to intermediate-risk disease, older patients, or those with other medical comorbidities as it is generally better-tolerated and has a good safety profile with the longest follow up. Affordability is key especially when treatment may continue throughout life. Imatinib still remains the most frequently used TKI around the world for various reasons, particularly because of lower cost, greater access, and familiarity. Regardless of which TKI is used as initial therapy, appropriate patient adherence, close monitoring of disease response, swift change in treatment for those who fail to meet treatment milestones and close monitoring for adverse effects is necessary to ensure treatment success.

\section{AUTHOR CONTRIBUTIONS}

$\mathrm{XB}$ and SR conducted research and drafted and revised this article. GK provided critical revision and final editing support. All authors contributed to the article and approved the submitted version.
CML: A Systematic Review and Meta-Analysis. Blood Adv (2020) 4(12):272335. doi: 10.1182/bloodadvances.2019001329

11. Hughes TP, Hochhaus A, Branford S, Muller MC, Kaeda JS, Foroni L, et al. Long-Term Prognostic Significance of Early Molecular Response to Imatinib in Newly Diagnosed Chronic Myeloid Leukemia: An Analysis From the International Randomized Study of Interferon and STI571 (IRIS). Blood (2010) 116(19):3758-65. doi: 10.1182/blood-2010-03-273979

12. Branford S, Kim DW, Soverini S, Haque A, Shou Y, Woodman RC, et al. Initial Molecular Response at 3 Months may Predict Both Response and Event-Free Survival at 24 Months in Imatinib-Resistant or -Intolerant Patients With Philadelphia Chromosome-Positive Chronic Myeloid Leukemia in Chronic Phase Treated With Nilotinib. J Clin Oncol (2012) 30(35):4323-9. doi: 10.1200/JCO.2011.40.5217

13. Jabbour E, Kantarjian H, O'Brien S, Shan J, Quintas-Cardama A, Faderl S, et al. The Achievement of an Early Complete Cytogenetic Response is a Major Determinant for Outcome in Patients With Early Chronic Phase Chronic Myeloid Leukemia Treated With Tyrosine Kinase Inhibitors. Blood (2011) 118 (17):4541-6; quiz 4759. doi: 10.1182/blood-2011-04-348110

14. Hochhaus A, Baccarani M, Silver RT, Schiffer C, Apperley JF, Cervantes F, et al. European LeukemiaNet 2020 Recommendations for Treating Chronic Myeloid Leukemia. Leukemia (2020) 34(4):966-84. doi: 10.1038/s41375-0200776-2

15. Neelakantan P, Gerrard G, Lucas C, Milojkovic D, May P, Wang L, et al. Combining BCR-ABL1 Transcript Levels at 3 and 6 Months in Chronic Myeloid Leukemia: Implications for Early Intervention Strategies. Blood (2013) 121(14):2739-42. doi: 10.1182/blood-2012-11-466037

16. Hanfstein B, Muller MC, Hehlmann R, Erben P, Lauseker M, Fabarius A, et al. Early Molecular and Cytogenetic Response is Predictive for Long-Term Progression-Free and Overall Survival in Chronic Myeloid Leukemia (CML). Leukemia (2012) 26(9):2096-102. doi: 10.1038/leu.2012.85

17. Hughes TP, Saglio G, Kantarjian HM, Guilhot F, Niederwieser D, Rosti G, et al. Early Molecular Response Predicts Outcomes in Patients With Chronic Myeloid Leukemia in Chronic Phase Treated With Frontline Nilotinib or Imatinib. Blood (2014) 123(9):1353-60. doi: 10.1182/blood-2013-06-510396

18. Marin D, Ibrahim AR, Lucas C, Gerrard G, Wang L, Szydlo RM, et al. Assessment of BCR-ABL1 Transcript Levels at 3 Months is the Only Requirement for Predicting Outcome for Patients With Chronic Myeloid Leukemia Treated With Tyrosine Kinase Inhibitors. J Clin Oncol (2012) 30 (3):232-8. doi: 10.1016/j.yonc.2012.07.031 
19. Jabbour E, Kantarjian HM, Saglio G, Steegmann JL, Shah NP, Boque C, et al. Early Response With Dasatinib or Imatinib in Chronic Myeloid Leukemia: 3Year Follow-Up From a Randomized Phase 3 Trial (DASISION). Blood (2014) 123(4):494-500. doi: 10.1182/blood-2013-06-511592

20. Cortes J, Rea D, Lipton JH. Treatment-Free Remission With First- and Second-Generation Tyrosine Kinase Inhibitors. Am J Hematol (2019) 94 (3):346-57. doi: 10.1002/ajh.25342

21. Rea D. Handling Challenging Questions in the Management of Chronic Myeloid Leukemia: When is it Safe to Stop Tyrosine Kinase Inhibitors? Blood Adv (2020) 4(21):5589-94. doi: 10.1182/bloodadvances.2020002538

22. Hehlmann R, Muller MC, Lauseker M, Hanfstein B, Fabarius A, Schreiber A, et al. Deep Molecular Response is Reached by the Majority of Patients Treated With Imatinib, Predicts Survival, and is Achieved More Quickly by Optimized High-Dose Imatinib: Results From the Randomized CML-Study IV. J Clin Oncol (2014) 32(5):415-23. doi: 10.1200/JCO.2013.49.9020

23. Kantarjian HM, Hughes TP, Larson RA, Kim DW, Issaragrisil S, le Coutre P, et al. Long-Term Outcomes With Frontline Nilotinib Versus Imatinib in Newly Diagnosed Chronic Myeloid Leukemia in Chronic Phase: ENESTnd 10-Year Analysis. Leukemia (2021) 35(2):440-53. doi: 10.1038/s41375-02001111-2

24. Hochhaus A, Saglio G, Hughes TP, Larson RA, Kim DW, Issaragrisil S, et al. Long-Term Benefits and Risks of Frontline Nilotinib vs Imatinib for Chronic Myeloid Leukemia in Chronic Phase: 5-Year Update of the Randomized ENESTnd Trial. Leukemia (2016) 30(5):1044-54. doi: 10.1038/leu.2016.5

25. O’Brien SG, Guilhot F, Larson RA, Gathmann I, Baccarani M, Cervantes F, et al. Imatinib Compared With Interferon and Low-Dose Cytarabine for Newly Diagnosed Chronic-Phase Chronic Myeloid Leukemia. N Engl J Med (2003) 348(11):994-1004. doi: 10.1056/NEJMoa022457

26. Cortes JE, Saglio G, Kantarjian HM, Baccarani M, Mayer J, Boque C, et al. Final 5-Year Study Results of DASISION: The Dasatinib Versus Imatinib Study in Treatment-Naive Chronic Myeloid Leukemia Patients Trial. J Clin Oncol (2016) 34(20):2333-40. doi: 10.1200/JCO.2015.64.8899

27. Brümmendorf TH, Cortes JE, Milojkovic D, Gambacorti-Passerini C, Clark RE, le Coutre PD, et al. Bosutinib (BOS) Versus Imatinib for Newly Diagnosed Chronic Phase (CP) Chronic Myeloid Leukemia (CML): Final 5Year Results From the Bfore Trial. Blood (2020) 136(Supplement 1):41-2.
28. Douxfils J, Haguet H, Mullier F, Chatelain C, Graux C, Dogne JM. Association Between BCR-ABL Tyrosine Kinase Inhibitors for Chronic Myeloid Leukemia and Cardiovascular Events, Major Molecular Response, and Overall Survival: A Systematic Review and Meta-Analysis. JAMA Oncol (2016) 2(5):625-32. doi: 10.1001/jamaoncol.2015.5932

29. Yamamoto C, Nakashima H, Ikeda T, Kawaguchi SI, Toda Y, Ito S, et al. Analysis of the Cost-Effectiveness of Treatment Strategies for CML With Incorporation of Treatment Discontinuation. Blood Adv (2019) 3(21):326677. doi: 10.1182/bloodadvances. 2019000745

30. Lyman GH, Henk HJ. Association of Generic Imatinib Availability and Pricing With Trends in Tyrosine Kinase Inhibitor Use in Patients With Chronic Myelogenous Leukemia. JAMA Oncol (2020) 6(12):1969-71. doi: 10.1001/jamaoncol.2020.4660

31. Cornelison AM, Kantarjian H, Cortes J, Jabbour E. Outcome of Treatment of Chronic Myeloid Leukemia With Second-Generation Tyrosine Kinase Inhibitors After Imatinib Failure. Clin Lymphoma Myeloma Leuk (2011) 11 Suppl 1:S101-10. doi: 10.1016/j.clml.2011.02.009

Conflict of Interest: The authors declare that the research was conducted in the absence of any commercial or financial relationships that could be construed as a potential conflict of interest.

The handling editor declared a shared affiliation with the authors at the time of review.

Publisher's Note: All claims expressed in this article are solely those of the authors and do not necessarily represent those of their affiliated organizations, or those of the publisher, the editors and the reviewers. Any product that may be evaluated in this article, or claim that may be made by its manufacturer, is not guaranteed or endorsed by the publisher.

Copyright (c) $2021 \mathrm{Bi}$, Ramanathan and Keiffer. This is an open-access article distributed under the terms of the Creative Commons Attribution License (CC BY). The use, distribution or reproduction in other forums is permitted, provided the original author(s) and the copyright owner(s) are credited and that the original publication in this journal is cited, in accordance with accepted academic practice. No use, distribution or reproduction is permitted which does not comply with these terms. 